\title{
A Case of Familial Spondyloenchondrodysplasia with Immune Dysregulation Masquerading as Moyamoya Syndrome
}

\author{
Jin Sook Lee ${ }^{a}$, Byung Chan Lim ${ }^{b}$ \\ Ki Joong Kim ${ }^{b}$, Seung-Ki Kim ${ }^{c}$ \\ Ok-Hwa Kim ${ }^{\mathrm{d}}$, Joong Gon Kim ${ }^{\mathrm{e}}$ \\ Sung-Gyoo Park', Tae-Joon $\mathrm{Cho}^{\mathrm{g}}$ \\ Jong-Hee Chae ${ }^{b}$ \\ aDepartment of Pediatrics, \\ Department of Genome Medicine and \\ Science, Gil Medical Center \\ Gachon University College of Medicine, \\ Incheon, Korea \\ ${ }^{b}$ Department of Pediatrics, \\ Pediatric Clinical Neuroscience Center \\ Seoul National University \\ Children's Hospital, \\ Seoul National University \\ College of Medicine, Seoul, Korea \\ 'Division of Pediatric Neurosurgery, \\ Seoul National University \\ Children's Hospital, \\ Seoul National University \\ College of Medicine, Seoul, Korea \\ dDepartment of Radiology, \\ Woorisoa Children's Hospital, \\ Seoul, Korea \\ eDepartment of Pediatrics, \\ Seoul National University \\ Children's Hospital, \\ Seoul National University \\ College of Medicine, Seoul, Korea \\ ${ }^{f}$ Cell Logistics Research Center and \\ School of Life Sciences, Gwangju Institute \\ of Science and Technology, Gwangju, \\ Korea \\ ${ }^{9}$ Division of Pediatric Orthopaedics, \\ Seoul National University \\ Children's Hospital, \\ Seoul National University \\ College of Medicine, Seoul, Korea
}

Received January 25, 2019

Revised March 1,2019

Accepted March 1, 2019

\section{Correspondence}

Jong-Hee Chae, $\mathrm{MD}, \mathrm{PhD}$

Department of Pediatrics,

Pediatric Clinical Neuroscience Center,

Seoul National University

Children's Hospital, Seoul National

University College of Medicine,

101 Daehak-ro, Jongno-gu,

Seoul 03080, Korea

Tel $+82-2-2072-3622$

Fax +82-2-743-3455

E-mail chaeped1@snu.ac.kr
Dear Editor,

Spondyloenchondrodysplasia with immune dysregulation (SPENCDI; OMIM\#607944) caused by ACP5 mutations is a rare skeletal dysplasia that was originally described with radiolucent spondylar and metaphyseal lesions. ${ }^{1}$ Neurological involvement-including spasticity, developmental delay, intracranial calcification, and leukodystrophy_has subsequently been reported. ${ }^{2,3}$ Moreover, immune dysregulations such as autoimmunity or immune deficiencies have also been documented. ${ }^{4}$ Systemic lupus erythematous (SLE) is also reportedly associated with SPENCDI. ${ }^{3}$ Here we report two siblings with $A C P 5$ variants presenting with moyamoya syndrome (MMS) that progressed to SLE.

A 3-year-old boy was admitted for recurrent transient ischemic attacks of monoplegia or hemiplegia on the right or left side, which usually improved within a few hours. Brain MRI and angiography revealed bilateral middle and anterior cerebral arterial stenoses with collateral circulation (Fig. 1A). His developmental milestones were within the normal range for age. No skin rash, oral lesions, or arthritis were noted. The findings of laboratory studies including an immunological workup were normal. He underwent left encephalogaleoperiosteal synangiosis with encephaloduroarteriosynangiosis (EDAS) operation, followed 1 month later by a right EDAS operation. Thereafter, multiple burr-hole trephinations were performed on both occipital areas. However, even after revascularization bypass surgery he still complained of transient ischemic attacks, involuntary choreoathetoid movements, and headache. At the age of 6 years he was admitted for fever of unknown origin. Skin lesions suggestive of Raynaud's phenomenon developed (Fig. 1F). Together with leukopenia, antinuclear antibody at 1:160 (homogeneous pattern), anti-ds DNA at $93 \mathrm{IU} / \mathrm{mL}$ (normal, $0-10$ $\mathrm{IU} / \mathrm{mL}$ ), positivity for anticardiolipin antibody IgM (25.8 MPL units) (normal, $<20 \mathrm{MPL}$ units), and C3/C4 levels of 58/9 mg/dL (normal, 70-150/10-35 mg/dL) were found. A renal biopsy was performed because of his persistent hematuria, which showed diffuse proliferative glomerulonephritis, and so he was diagnosed with SLE and lupus nephritis.

Skeletal surveys performed at 9 years of age due to chronic pain in the extremities revealed platyspondyly and metaphyseal dysplasia, suggesting spondyloenchondrodysplasia (Fig. 1CE). Follow-up neuroimaging findings at the age of 15 years showed an old infarction with cerebromalacia in the left parieto-occipital and both frontal areas, and total obstruction of both the middle and posterior cerebral arteries and distal internal carotid arteries with neovascularization. His older sister developed similar neurological symptoms: MMS (Fig. 1B) and SLE at 8 and 11 years of age, respectively. These skeletal findings prompted us to perform a skeletal dysplasia gene panel, which confirmed compound heterozygous ACP5 variants in the siblings [OMIM*171640, NM_001111035; c.136C >T (p.R46W) and c.449T>A (p. V150E)], which have been reported previously. ${ }^{5}$ The unaffected parents were heterozygous

@ This is an Open Access article distributed under the terms of the Creative Commons Attribution Non-Commercial License (https://creativecommons.org/licenses/by-nc/4.0) which permits unrestricted non-commercial use, distribution, and reproduction in any medium, provided the original work is properly cited. 

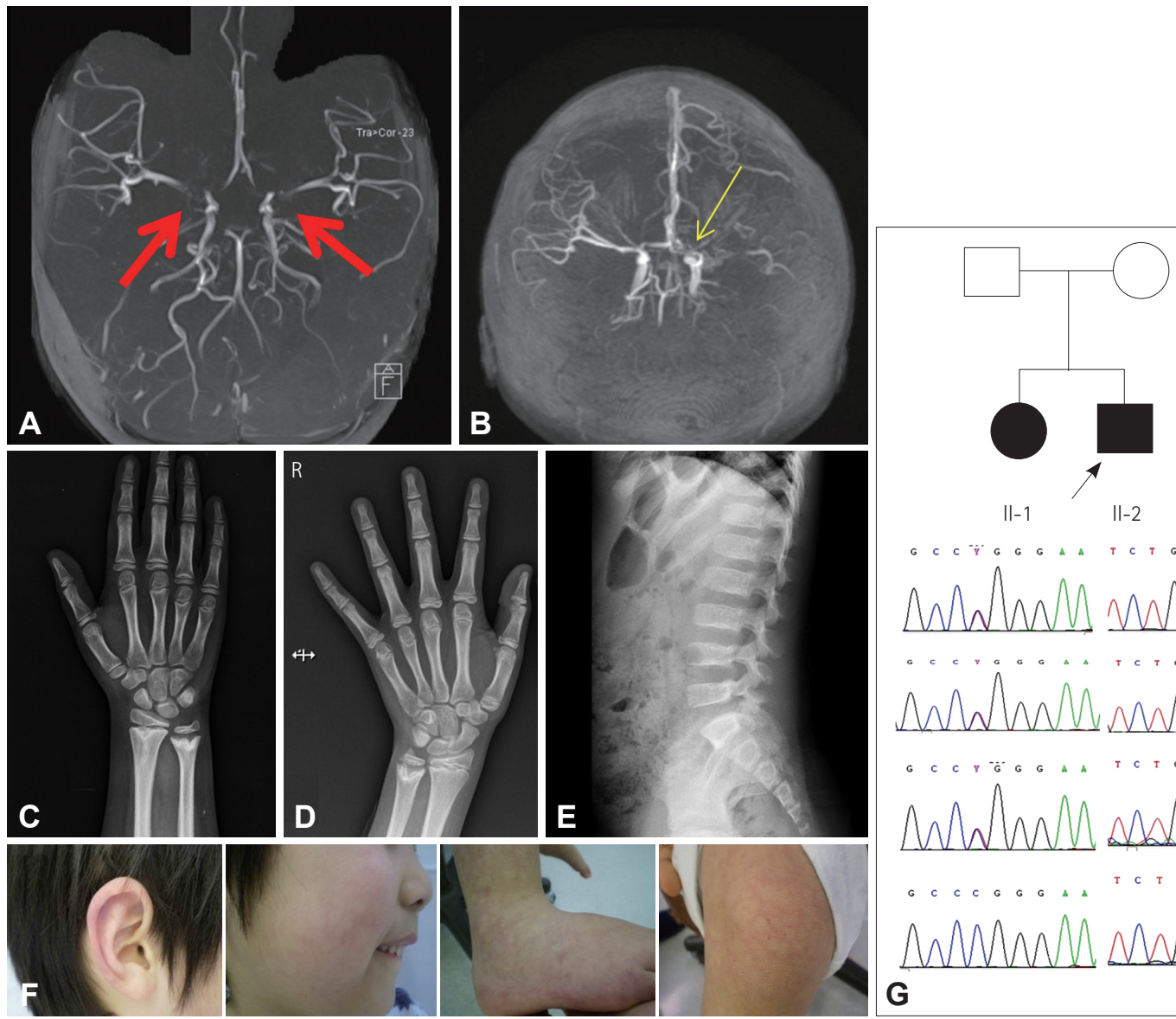

$\|-1$

$\|-2$

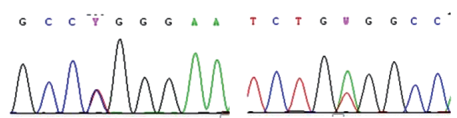

I-2

p.R46W/p.V150E

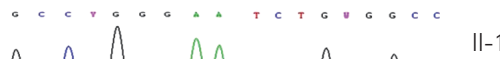

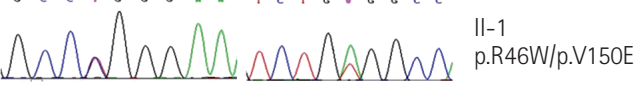

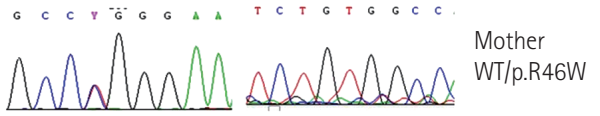

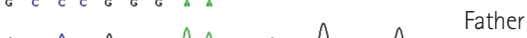

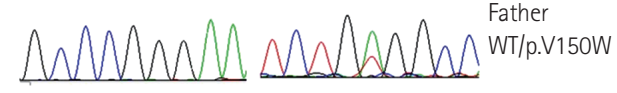

G

Fig. 1. Radiology, photograph and Sanger sequencing. Magnetic resonance angiography images of the brain show bilateral stenosis/occlusion (red arrows) of the proximal middle and anterior cerebral arteries in the proband (II-2) (A) and complete occlusion (yellow arrow) of the left middle and anterior cerebral arteries and focal narrowing of the right proximal middle cerebral artery in his sister (II-1) (B). Radiographic photographs of the proband show bilateral metaphyseal irregularities with cupped and enchondromata-like streaks in the radius and ulna (C and D), and generalized platyspondyly of the vertebral bodies with a rectangular shape (E). Skin lesions of the proband were suggestive of Raynaud's phenomenon (F). Pedigrees and Sanger sequencing revealed compound heterozygous variants of $A C P 5$ in both affected siblings: c.136C>T (p.R46W) and c.449T>A (p.V150E). The black arrow indicates the proband (II-2) (G). WT: wild type.

carriers of each variant. There were increased levels of interferon (IFN)- $\alpha$ and $-\beta$ as well as dominant Th 1 and Th17 responses. Serum cytokines were measured with the Cytokine Bead Array (BD Biosciences, Franklin Lakes, NJ, USA) using the Canto II flow cytometer (BD Biosciences), and serum IFN levels were analyzed by an enzyme-linked immunosorbent assay (eBioscience/Thermo Fisher Scientific, Waltham, MA, USA) according to the manufacturer's instructions (Supplementary Material and Supplementary Fig. 1 in the online-only Data Supplement).

There have been few reports of comorbid angiopathy in patients with SPENCDI, ${ }^{4,6}$ and no reports on SLE presenting with MMS in patients with SPENCDI. The present study has expanded the neurological phenotypic spectrum of SPENCDI, by adding lupus with MMS. This information will help to improve our understanding of the pathophysiology of MMS associated with autoimmune diseases, in combination with sev- eral previous reports suggesting that immune responses can contribute to the development of MMS. ${ }^{7,8}$ Therefore, SPEN$\mathrm{CDI}$ should be considered as a possible underlying cause in patients presenting with early-onset or familial SLE with angiopathies, even when they show only mild skeletal radiological characteristics. Conversely, early neuroimaging screening or follow-up might be needed to prevent cerebrovasculopathy-related neurological abnormalities in SPENCDI patients.

\section{Supplementary Materials}

The online-only Data Supplement is available with this article at https://doi.org/10.3988/jen.2019.15.3.407.

\section{Conflicts of Interest}

The authors have no potential conflicts of interest to disclose.

\section{Acknowledgements}

This research was supported by a grant of the Korea Health Technology 
R\&D Project through the Korea Health Industry Development Institute (KHIDI), funded by the Ministry of Health \& Welfare, Republic of Korea (Grant No. HI16C1986).

\section{REFERENCES}

1. Schorr S, Legum C, Ochshorn M. Spondyloenchondrodysplasia. Enchondromatomosis with severe platyspondyly in two brothers. Radiology 1976;118:133-139.

2. Frydman M, Bar-Ziv J, Preminger-Shapiro R, Brezner A, Brand N, Ben-Ami T, et al. Possible heterogeneity in spondyloenchondrodysplasia: quadriparesis, basal ganglia calcifications, and chondrocyte inclusions. Am J Med Genet 1990;36:279-284.

3. Briggs TA, Rice GI, Adib N, Ades L, Barete S, Baskar K, et al. Spondyloenchondrodysplasia due to mutations in ACP5: a comprehensive survey. J Clin Immunol 2016;36:220-234.
4. Renella R, Schaefer E, LeMerrer M, Alanay Y, Kandemir N, Eich G, et al. Spondyloenchondrodysplasia with spasticity, cerebral calcifications, and immune dysregulation: clinical and radiographic delineation of a pleiotropic disorder. Am J Med Genet A 2006;140:541-550.

5. Bae JS, Kim NK, Lee C, Kim SC, Lee HR, Song HR, et al. Comprehensive genetic exploration of skeletal dysplasia using targeted exome sequencing. Genet Med 2016;18:563-569.

6. Navarro V, Scott C, Briggs TA, Barete S, Frances C, Lebon P, et al. Two further cases of spondyloenchondrodysplasia (SPENCD) with immune dysregulation. Am J Med Genet A 2008;146A:2810-2815.

7. Wang R, Xu Y, Lv R, Chen J. Systemic lupus erythematosus associated with Moyamoya syndrome: a case report and literature review. Lupus 2013;22:629-633.

8. Jeong HC, Kim YJ, Yoon W, Joo SP, Lee SS, Park YW. Moyamoya syndrome associated with systemic lupus erythematosus. Lupus 2008;17: 679-682. 